\title{
Cryotherapy for Intra- and Perianal High-Grade Squamous Intraepithelial Lesions in HIV-Positive Men who have Sex with Men
}

\author{
Matthijs L. Siegenbeek van Heukelom ${ }^{1,2,3}$ (D) Karien C. M. Gosens ${ }^{2,3}$ • \\ Jan M. Prins ${ }^{1,2} \cdot$ Henry J. C. de Vries ${ }^{2,3,4}$
}

Published online: 10 July 2017

(C) The Author(s) 2017. This article is an open access publication

\begin{abstract}
Background Available treatment options for anal highgrade squamous intraepithelial lesions (HSIL) in HIVpositive men who have sex with men (MSM) are limited by low response rates and frequent recurrences. Cryotherapy is an established therapeutic option for several pre-malignant skin disorders.

Methods This retrospective, non-randomized study included HIV-positive MSM who received intra- and/or perianal HSIL cryotherapy treatment between 30 December 2008 and 23 April 2015. Cryotherapy was applied in sessions 4-6 weeks apart for a maximum of five sessions. Patients received a follow-up high-resolution anoscopy (HRA) to assess treatment response. Complete and partial treatment responders were followed-up after 6 months and then every 6-12 months to investigate recurrent HSILs.

Results Of 64 patients [median age 48 years; interquartile range (IQR) 42-56] included in the study, six were lost to follow-up. In total, 35 (60\%) of 58 patients responded to treatment. Of 64 patients, 31 (48\%) reported one or more side effects, of which anal pain or tenderness and mild
\end{abstract}

Matthijs L. Siegenbeek van Heukelom

m.l.vanheukelom@amc.uva.nl

1 Department of Internal Medicine, Division of Infectious

Diseases, Academic Medical Center, Room F4-106,

Meibergdreef 9, 1105 AZ Amsterdam, The Netherlands

2 Center for Infection and Immunology Amsterdam (CINIMA), Academic Medical Center, 1105 AZ Amsterdam, The Netherlands

3 Department of Dermatology, Academic Medical Center, 1105 AZ Amsterdam, The Netherlands

4 STI Outpatient Clinic, Public Health Service of Amsterdam (GGD Amsterdam), 1018 WT Amsterdam, The Netherlands blood loss were reported most frequently. A total of 19 patients who responded to cryotherapy were adequately followed-up for over 18 months, of whom 13 (68\%) had recurrent HSILs.

Conclusion Cryotherapy is capable of clearing HSIL in HIV-positive MSM, and treatment success rates are comparable with those reported for current treatment modalities. The treatment is well tolerated, and side effects are relatively mild. Future studies should therefore compare the efficacy and tolerability of cryotherapy with those of current treatment modalities in randomized controlled trials.

\section{Key points}

Cryotherapy is capable of clearing high-grade squamous intraepithelial lesions in HIV-positive men who have sex with men (MSM).

Side effects following cryotherapy for high-grade squamous intraepithelial lesions are mild.

Recurrences of high-grade squamous intraepithelial lesions are frequent in HIV-positive MSM treated with cryotherapy.

\section{Introduction}

HIV-positive men who have sex with men (MSM) are at risk for developing anal squamous cell carcinoma. Incidence rates have increased from 13-37 per 100,000 person- 
years in the pre-combination antiretroviral therapy (cART) era to $65-109$ per 100,000 person-years in the cART era [1]. Anal intraepithelial neoplasia (AIN) is a precursor stage of anal squamous cell carcinoma and follows infection with human papillomavirus (HPV). High-resolution anoscopy (HRA)-guided biopsies are the gold standard for identifying AIN, and the prevalence of low-grade squamous intraepithelial lesions (LSILs or AIN 1) and highgrade squamous intraepithelial lesions (HSILs or AIN 2-3) is high in HIV-positive MSM: 31.3 and $29.1 \%$, respectively [1]. Treatment of HSIL in HIV-positive MSM is highly recommended to prevent progression towards invasive disease [2]. Large randomized controlled trials are currently investigating the natural history of AIN [3-5].

Several treatment modalities for the treatment of HSIL have been described. Treatment varies from targeted destruction of HSILs using infrared coagulation, electrocautery, cryosurgery, or surgical excision to immunomodulation and photodynamic therapy, but with little consensus between the guidelines [6-8]. Electrocautery is currently the treatment of choice for intra-anal HSIL in many centers, but response rates with all HSIL treatment modalities are disappointingly low and recurrence rates are high $[9,10]$. Therefore, new treatment modalities or techniques should be considered.

Cryotherapy is an old technique [11] that was first introduced in dermatology in 1899 [12] and is currently still widely used for treatment of HPV-induced pre-malignant and malignant skin disorders such as HPV-associated warts, actinic keratosis, and basal cell carcinoma [13-15]. Anogenital condylomata are commonly treated with liquid nitrogen because it is easily applied, is cheap, leaves no scar tissue when applied correctly, and achieves acceptable clearance rates [16].

In this retrospective study, we describe the response and recurrence rates of cryotherapy for intra- and perianal HSIL in HIV-positive MSM.

\section{Materials and Methods}

\subsection{Patients}

We studied HIV-positive MSM aged $\geq 30$ years who underwent AIN screening with HRA and were diagnosed with intra- and/or perianal HSIL, as described by Richel et al. [17] (Fig. 1). In short, screening was performed by one of three expert anoscopists (having performed more than 200 HRAs each) and consisted of a digital rectal exam followed by peri- and intra-anal inspection with a colposcope (ZEISS opmi pico surgical microscope) after repeated application of acetic acid (3\% solution) and staining with Lugol's iodine when indicated. All lesions suspicious

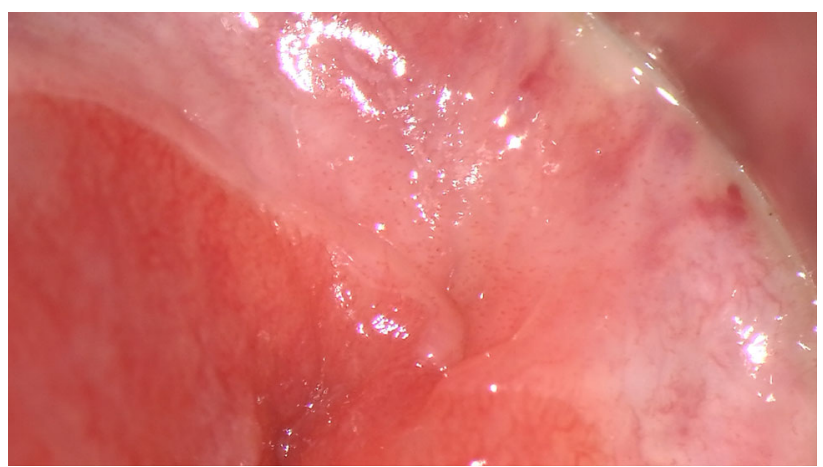

Fig. 1 Intra-anal high-grade squamous intraepithelial lesion precryotherapy treatment

for AIN were biopsied and graded by one of two pathologists who specialized in squamous intraepithelial lesions. The extent of intra-anal HSIL was analyzed by describing the number of quadrants containing HSIL disease. No anal cytology was performed in any of the patients. Patients diagnosed with intra- and/or perianal HSIL were offered standard electrocoagulation therapy and cryotherapy, with the latter introduced as a new treatment modality with unknown efficacy.

\subsection{Cryotherapy}

Patients scheduled for cryotherapy at the Academic Medical Center (Amsterdam, the Netherlands) between 30 December 2008 and 23 April 2015 attended a maximum of five treatment visits, each visit 4-6 weeks apart. Treatment for intraand perianal lesions consisted of office-based HRA-guided liquid nitrogen application in three freeze-thaw cycles using a spray gun with a standard $0.56 \mathrm{~mm}$ aperture, spraying $10 \mathrm{~s}$ per lesion (Fig. 2). At each treatment visit, self-reported side effects were noted and inquiries were made regarding blood loss and pain that may have resulted from the previous treatment, followed by inspection with HRA and guided cryotherapy for remaining HSIL lesions. If no lesions were seen, treatment response was defined as a complete response

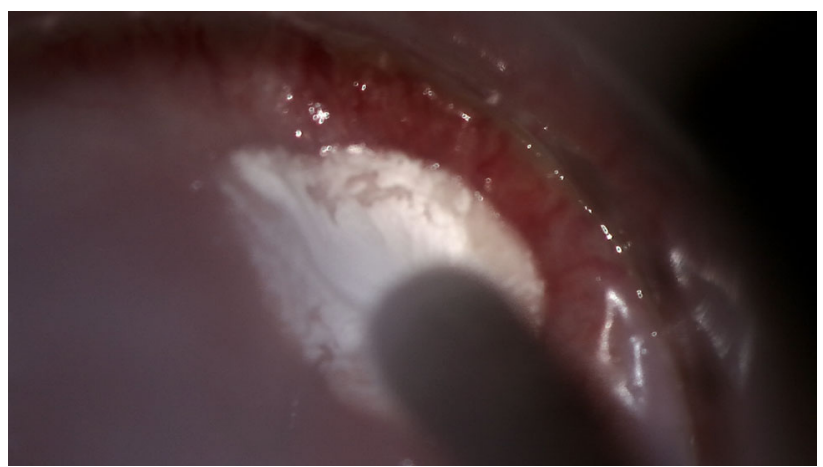

Fig. 2 Intra-anal high-grade squamous intraepithelial lesion postcryotherapy treatment 
(CR), no further treatments were given, and a 6-month follow-up visit was scheduled.

Treatment response was assessed 4-6 weeks after the final treatment. During this session, the intra- and perianal region was again inspected and all remaining lesions biopsied. If no lesions were seen, treatment response was defined as $\mathrm{CR}$ and no biopsies were taken. If histological resolution of all AIN lesions was achieved, treatment response was also defined as CR. If all HSIL lesions had regressed to at least LSIL (AIN 1), treatment response was defined as partial response (PR), and, if invasion had occurred, response was defined as progressive disease. In all other cases, the outcome was defined as stable disease (SD), which was analyzed in more detail to distinguish between persistent HSIL (in the same location) or metachronous HSIL (in a new location). Patients with CR or PR were scheduled for a follow-up HRA in 6 months. Patients with SD were again offered treatment with one of the remaining treatment modalities described, and patients with progressive disease were referred for oncologic treatment options.

Patients who received cryotherapy and completed at least one follow-up visit were included in the analyses. Patients lost to follow-up before a treatment response could be evaluated were excluded from the analyses. Patients who were lost to follow-up after the treatment response was evaluated, and patients who participated in a quadrivalent HPV vaccination trial (VACCAIN-P study) were excluded from recurrence rate analyses.

\subsection{Statistical Analysis}

Potential risk factors for cryotherapy treatment successnadir cluster of differentiation (CD)-4 cell count and ever smoking-were tested using univariable logistic regression analysis. Variables were considered significantly associated when the $p$ value was $<0.05$. Statistical analyses were performed using SPSS software (version 23.0.3; IBM, Armonk, NY, USA). Data were expressed as medians with interquartile ranges.

\section{Results}

\subsection{Patient Characteristics}

Between 30 December 2008 and 23 April 2015, 64 patients were treated with cryotherapy and included in the present study. Their median age was 48 years, the median $\mathrm{CD}^{+}{ }^{+} \mathrm{T}$-cell count during treatment was 640 cells $/ \mu \mathrm{L}$, and 60 of 64 patients (94\%) were using cART. Of the patients receiving cryotherapy, $52(81 \%)$ received it for intra-anal HSIL only, one $(2 \%)$ for perianal HSIL only, and $11(17 \%)$ for both intra- and perianal HSIL. Baseline characteristics are listed in Table 1.
Table 1 Characteristics of HIV-positive men who have sex with men with anal high-grade squamous intraepithelial lesions treated with cryotherapy

\begin{tabular}{ll}
\hline Characteristic & Data $^{\mathrm{a}}$ \\
\hline Patients $(N)$ & 64 \\
Age (years) & $48(42-56)$ \\
Patients using cART at first cryotherapy & $60 / 64(94)$ \\
Nadir CD4 ${ }^{+}$cell count $\left(\right.$cells $\left.\mu \mathrm{L}^{-1}\right)$ & $290(150-410)$ \\
Current CD4 ${ }^{+}$cell count (cells $\left.\mu \mathrm{L}^{-1}\right)$ & $640(480-800)$ \\
Ever smoker & $35 / 59(59)$ \\
$\quad$ Pack-years & $5(0-20)$ \\
Number of biopsies & $3(2-4)$ \\
Perianal HSIL & $12 / 64(19)$ \\
Intra-anal HSIL & $63 / 64(98)$ \\
One quadrant & $45 / 63(71)$ \\
Two quadrants & $12 / 63(19)$ \\
Three quadrants & $5 / 63(8)$ \\
Four quadrants & $1 / 63(2)$ \\
\hline
\end{tabular}

$c A R T$ combination antiretroviral therapy, $C D$ cluster of differentiation, HSIL high-grade squamous intraepithelial lesions

${ }^{a}$ Data are presented as median (interquartile range) or $n / N(\%)$ unless otherwise indicated

\subsection{Treatment Response}

Six of 64 patients were lost to follow-up before a treatment response could be evaluated (Fig. 3). Of 58 patients, 35 $(60 \%)$ had either a CR $(n=20)$ or a PR $(n=15)$ (Table 2). Of 12 patients treated for perianal HSIL, four (33\%) had either a CR $(n=1)$ or a PR $(n=3)$. Of 57 patients treated for intra-anal HSIL, $34(60 \%)$ had either a CR $(n=19)$ or a PR $(n=15)$. In total, 27 of 40 patients $(68 \%)$ with intra-anal HSIL in one quadrant, 6 of $12(50 \%)$ with HSIL in two quadrants, and one of four (25\%) with HSIL in three quadrants had either a CR or a PR. The only patient with HSIL in four quadrants had SD. Of the 23 patients with SD, $11(40 \%)$ had persistent HSIL, seven (30\%) showed only metachronous HSIL, and five $(21 \%)$ showed both persistent and metachronous HSIL after treatment. None of the patients had progressive disease during the course of this study.

Nadir CD4 cell count was not significantly associated with CR, PR, or SD. Ever smoking was not significantly associated with $\mathrm{CR}(p=0.493)$ or PR $(p=0.087)$ but did significantly increase the odds of SD [odds ratio (OR) 0.26; 95\% confidence interval (CI) $0.078-0.871 ; p=0.029]$.

\subsection{Recurrence Rate}

Of the 35 patients with a CR or PR, 14 were screened and selected for the VACCAIN-P study at their first follow-up visit and were therefore excluded from further analyses. Of 
Fig. 3 Flowchart of treatment intervals and treatment results in HIV-positive men who have sex with men receiving cryotherapy as treatment for anal high-grade squamous intraepithelial lesions. $C R$ complete response, $L T F U$ lost to follow-up, $n$ number of study participants, $P R$ partial response, $S D$ stable disease, $T$ time in weeks

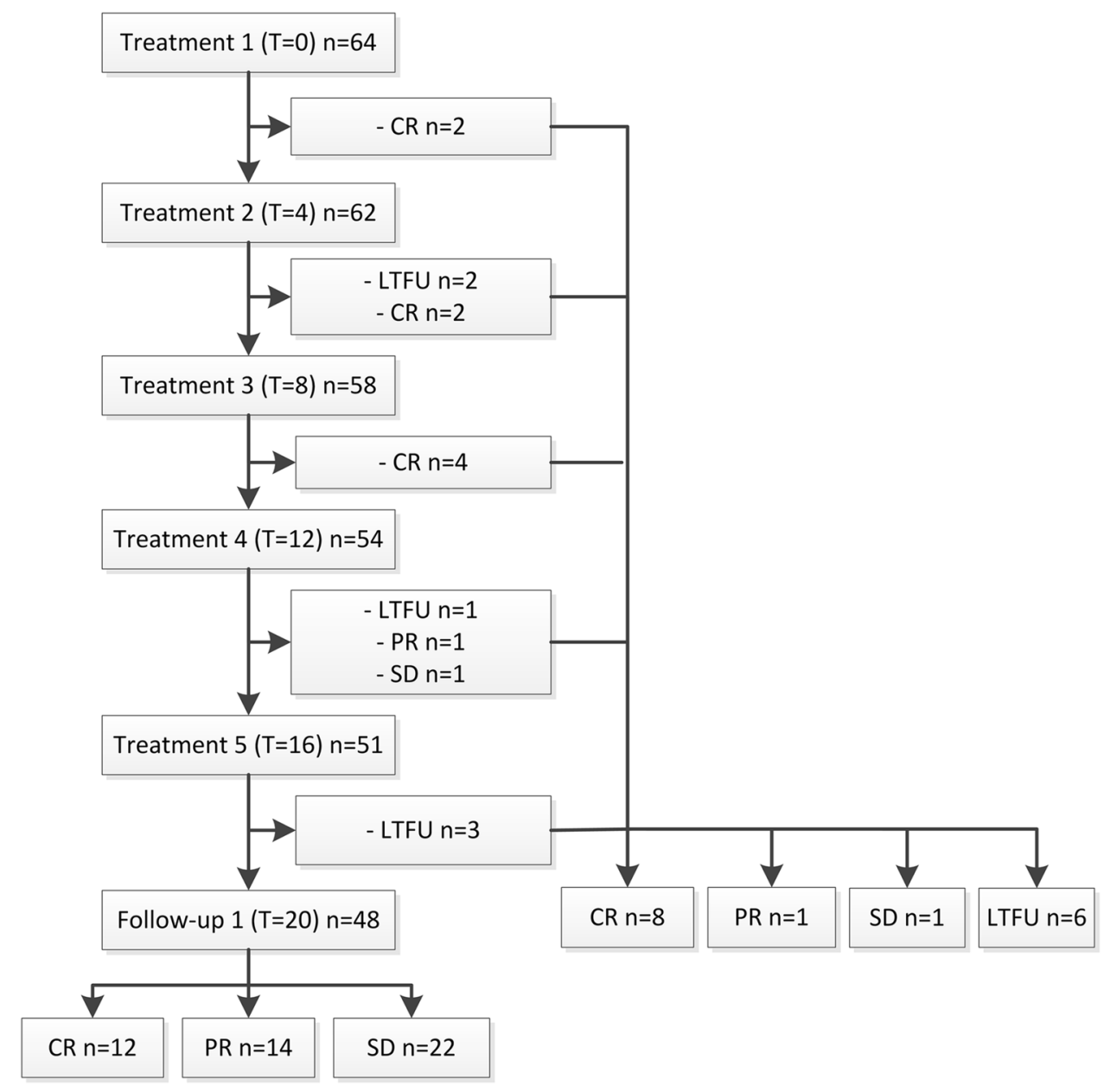

Table 2 Response to treatment of HIV-positive men who have sex with men with anal high-grade squamous intraepithelial lesions treated with cryotherapy

\begin{tabular}{llll}
\hline & CR & PR & SD \\
\hline $\begin{array}{l}\text { All HSIL } \\
\text { HSIL grading }\end{array}$ & $20 / 58(34)$ & $15 / 58(26)$ & $23 / 58(40)$ \\
$\quad$ AIN 2 & $12 / 34(35)$ & $10 / 34(29)$ & $12 / 34(35)$ \\
AIN 3 & $8 / 24(33)$ & $5 / 24(21)$ & $11 / 24(46)$ \\
Perianal HSIL & $1 / 12(8)$ & $3 / 12(25)$ & $8 / 12(66)$ \\
Intra-anal HSIL & $19 / 57(33)$ & $15 / 57(26)$ & $23 / 57(40)$ \\
Quadrants with intra-anal HSIL & & \\
$\quad$ One & $16 / 40(40)$ & $11 / 40(28)$ & $13 / 40(32)$ \\
Two & $3 / 12(25)$ & $3 / 12(25)$ & $6 / 12(50)$ \\
Three & 0 & $1 / 4(25)$ & $3 / 4(75)$ \\
Four & 0 & 0 & $1 / 1(100)$ \\
\hline
\end{tabular}

Data are presented as $n / N(\%)$

$A I N$ anal intraepithelial neoplasia, $C R$ complete response, $H S I L$ highgrade squamous intraepithelial lesions, $P R$ partial response, $S D$ stable disease the 21 remaining patients, 19 (seven CR, 12 PR) were adequately followed-up, meaning they received a follow-up HRA 6 months after their initial treatment response was evaluated. Of these 19 patients, seven (37\%; four CR and three PR) had an HSIL recurrence at first follow-up. Thereafter, 11 of the 12 remaining patients received a second follow-up visit in the next 6-19 months, and five (all PR) had a recurrence of HSIL. Of the remaining six patients (three CR, three PR), three had no HSIL recurrence at their third follow-up (one CR, two PR), one (CR) had recurrent HSIL and two were lost to follow-up (one CR, one PR). Overall, 13 of the 19 patients $(68 \%)$ who experienced a CR or a PR after cryotherapy, were not vaccinated against HPV, and had at least one follow-up visit after 6 months, had a documented HSIL recurrence within 18 months.

\subsection{Side Effects}

In total, 31 of 64 patients $(48 \%)$ reported one to three side effects during the cryotherapy treatment period: $19(30 \%)$ 
reported one side effect, nine (14\%) reported two, and three $(5 \%)$ reported three. Of the 64 patients, 20 (31\%) reported anal pain or tenderness during and/or following treatment, $14(22 \%)$ reported mild blood loss for a few days, four (6\%) experienced psychological problems (erectile dysfunction, stress, anxiety), and eight (13\%) reported other side effects (anal fissure, constipation, temporary fecal incontinence, diarrhea, hemorrhoid). None of the treated patients required additional medical attention because of side effects. One patient with a history of anal carcinoma discontinued treatment because of anal pain and psychological stress.

\section{Discussion}

In this study, we investigated cryotherapy for the treatment of HSIL in HIV-positive MSM. Cryotherapy resulted in a CR or PR in $60 \%$ of patients. Half of the patients reported side effects from cryotherapy, most of which were mild pain and blood loss.

The World Health Organization recommends cryotherapy for the treatment of cervical intraepithelial neoplasia (CIN) when the loop electrosurgical excision procedure (LEEP) is not available [18]. LEEP is more effective, as it allows the treating physician to remove the entire transitional zone in one procedure; however, the transitional zone of the anal canal is anatomically unfit for LEEP. Esophageal high-grade intraepithelial neoplasia at the pectinate line (Barrett's HG dysplasia) can be effectively and safely treated by endoscopic application of liquid nitrogen [19]. Studies showed high rates of clearance with endoscopic cryotherapy [19], but recurrence rates were higher than with photodynamic therapy or endoscopic mucosal resection [20].

Response rates for available HSIL treatment modalities (trichloroacetic acid [TCA], imiquimod, infrared coagulation, electrocautery) are similar to our rates for cryotherapy (61\% [21], 61\% [22], 64\% [23], and 69\% [9], respectively). However, if we observe the effect of cryotherapy on a lesional level in patients with SD, seven of 23 patients could be considered 'partial responders' because they developed metachronous lesions and all original HSIL lesions cleared; thus, the overall response rate would increase to 42 of 58 patients (72\%). In this small group of patients, univariate analysis indicated that ever smoking was positively associated with SD. Future studies with larger patient groups should perform multivariate analysis to assess whether smoking is a consistent risk factor for treatment failure. Cryotherapy for HSIL in one intra-anal quadrant showed a higher treatment response $(68 \%)$ than for HSIL in two quadrants (50\%) or three quadrants $(25 \%)$. For perianal HSIL, imiquimod treatment still shows a better treatment response than cryotherapy ( 91 vs. $36 \%$ ), making imiquimod the more effective choice for perianal HSIL [9].

Side effects from electrocautery, imiquimod, 5-fluorouracil, and cryotherapy were reported in $76,47,65$, and $50 \%$ of patients, respectively; however, this was not a randomized controlled study [9]. In the British Association of Dermatologists' guidelines for the management of Bowen's disease, Morton et al. [24] recommend cryotherapy as a generally good choice of treatment for single or multiple small $(<2 \mathrm{~cm})$, good healing lesions (refers to the clinician's perceived potential for good healing at the affected site). None of the possible complications following cryotherapy described by Morton et al. [24] (poor healing and hypopigmented scarring) were observed in our study.

The recurrence rate following a $\mathrm{CR}$ or $\mathrm{PR}$ after cryotherapy for HSIL appeared to be lower than after treatment with electrocautery, but follow-up visits in our study were less stringent than in prospective studies, and recurrence rates may therefore have been underestimated $[9,25]$.

To our knowledge, this is the first study to describe the treatment effects of cryotherapy for intra- and perianal HSIL in HIV-positive MSM. Although the retrospective, non-comparative design is a limitation of this study, this is a first step to finding a new possible treatment modality for HSIL. The inclusion of several patients with persistent $(n=2)$ or recurrent $(n=22)$ HSIL after electrocoagulation and other treatment options (5-fluorouracil, imiquimod, or TCA) could have introduced selection bias; therefore, the reported treatment response could underestimate the true potential of cryotherapy.

\section{Conclusion}

Cryotherapy is capable of clearing HSIL in HIV-positive MSM. Cryotherapy is well tolerated and side effects are common and mostly mild, but HSIL recurrences were frequent. A prospective, randomized study comparing electrocautery and cryotherapy should further investigate the efficacy and tolerability of cryotherapy as a new treatment modality for intra-anal HSIL.

Acknowledgements The authors are indebted to all study participants. We thank H. E. Nobel for providing excellent patient care and A. Henderiks for identifying study patients, both at the Academic Medical Center in Amsterdam, the Netherlands. They were not compensated for their contributions.

\section{Compliance with Ethical Standards}

Informed consent Not applicable. 
Ethics approval Not applicable.

Funding No sources of funding were used to conduct this study or prepare this manuscript.

Conflict of interest MLSH, KCMG, HJCV, and JMP have no conflicts of interest.

Open Access This article is distributed under the terms of the Creative Commons Attribution-NonCommercial 4.0 International License (http://creativecommons.org/licenses/by-nc/4.0/), which permits any noncommercial use, distribution, and reproduction in any medium, provided you give appropriate credit to the original author(s) and the source, provide a link to the Creative Commons license, and indicate if changes were made.

\section{References}

1. Machalek DA, Poynten M, Jin F, Fairley CK, Farnsworth A, Garland SM, et al. Anal human papillomavirus infection and associated neoplastic lesions in men who have sex with men: a systematic review and meta-analysis. Lancet Oncol. 2012;13(5):487-500. doi:10.1016/s1470-2045(12)70080-3.

2. Chin-Hong PV, Palefsky JM. Natural history and clinical management of anal human papillomavirus disease in men and women infected with human immunodeficiency virus. Clin Infect Dis. 2002;35(9):1127-34. doi:10.1086/344057.

3. Machalek DA, Grulich AE, Hillman RJ, Jin F, Templeton DJ, Tabrizi SN, et al. The Study of the Prevention of Anal Cancer (SPANC): design and methods of a three-year prospective cohort study. BMC Public Health. 2013;13:946. doi:10.1186/1471-245813-946.

4. AIDS Malignancy Consortium. Topical or ablatice treatment in preventing anal cancer in patients with HIV and anal high-grade squamous intraepithelial lesions (ANCHOR) [ClinicalTrials.gov identifier: NCT02135419]. US National Institutes of Health, ClinicalTrials.gov. https://clinicaltrials.gov/ct2/show/NCT02135 419. Accessed 4 July 2017.

5. The LOPAC Trial: Laser Ablation versus Observation to Prevent Anal Cancer. 2015. http://www.isrctn.com/ISRCTN14067023. Accessed 4 July 2017.

6. Alam NN, White DA, Narang SK, Daniels IR, Smart NJ. Systematic review of guidelines for the assessment and management of high grade anal intraepithelial neoplasia (AIN II/III). Colorectal Dis. 2016;18(2):135-46. doi:10.1111/codi.13215.

7. Macaya A, Munoz-Santos C, Balaguer A, Barbera MJ. Interventions for anal canal intraepithelial neoplasia. Cochrane Database Syst Rev. 2012;12:CD009244. doi:10.1002/14651858. CD009244.pub2.

8. Sendagorta E, Bernardino JI, Alvarez-Gallego M, Feito M, Feltes $\mathrm{R}$, Beato MJ, et al. Topical cidofovir to treat high-grade anal intraepithelial neoplasia in HIV-infected patients: a pilot clinical trial. AIDS. 2016;30(1):75-82. doi:10.1097/QAD.000000000000 0886.

9. Richel O, de Vries HJ, van Noesel CJ, Dijkgraaf MG, Prins JM. Comparison of imiquimod, topical fluorouracil, and electrocautery for the treatment of anal intraepithelial neoplasia in HIVpositive men who have sex with men: an open-label, randomised controlled trial. Lancet Oncol. 2013;14(4):346-53. doi:10.1016/ S1470-2045(13)70067-6.
10. Burgos J, Curran A, Landolfi S, Navarro J, Tallada N, Guelar A, et al. The effectiveness of electrocautery ablation for the treatment of high-grade anal intraepithelial neoplasia in HIV-infected men who have sex with men. HIV Med. 2016;17(7):524-31. doi:10.1111/hiv.12352.

11. Maranda E, Simmons BJ, Romanelli P. Cryotherapy: as ancient as the Pharaohs. JAMA Dermatol. 2016;152(6):730. doi:10.1001/ jamadermatol.2015.1616.

12. Cooper SM, Dawber RP. The history of cryosurgery. J R Soc Med. 2001;94(4):196-201.

13. Fathi R, Tsoukas MM. Genital warts and other HPV infections: established and novel therapies. Clin Dermatol. 2014;32(2):299-306. doi:10.1016/j.clindermatol.2013.08.014.

14. Gupta AK, Paquet M, Villanueva E, Brintnell W. Interventions for actinic keratoses. Cochrane Database Syst Rev. 2012;12:CD004415. doi:10.1002/14651858.CD004415.pub2.

15. Clark CM, Furniss M, Mackay-Wiggan JM. Basal cell carcinoma: an evidence-based treatment update. Am J Clin Dermatol. 2014;15(3):197-216. doi:10.1007/s40257-014-0070-z.

16. Lacey CJ. Therapy for genital human papillomavirus-related disease. J Clin Virol. 2005;32(Suppl 1):S82-90. doi:10.1016/j. jev.2004.10.020.

17. Richel O, Hallensleben ND, Kreuter A, van Noesel CJ, Prins JM, de Vries HJ. High-resolution anoscopy: clinical features of anal intraepithelial neoplasia in HIV-positive men. Dis Colon Rectum. 2013;56(11):1237-42. doi:10.1097/DCR.0b013e3182a53568.

18. World Health Organization. Treatment of cervical intraepithelial neoplasia 2-3 and adenocarcinoma in situ: cryotherapy, large loop excision of the transformation zone, and cold knife conization. WHO Guidelines. Geneva: WHO; 2014.

19. Shaheen NJ, Greenwald BD, Peery AF, Dumot JA, Nishioka NS, Wolfsen HC, et al. Safety and efficacy of endoscopic spray cryotherapy for Barrett's esophagus with high-grade dysplasia. Gastrointest Endosc. 2010;71(4):680-5. doi:10.1016/j.gie.2010. 01.018 .

20. Halsey KD, Chang JW, Waldt A, Greenwald BD. Recurrent disease following endoscopic ablation of Barrett's high-grade dysplasia with spray cryotherapy. Endoscopy. 2011;43(10):844-8. doi:10.1055/s-0030-1256649.

21. Singh JC, Kuohung V, Palefsky JM. Efficacy of trichloroacetic acid in the treatment of anal intraepithelial neoplasia in HIVpositive and HIV-negative men who have sex with men. J Acquir Immune Defic Syndr. 2009;52(4):474-9. doi:10.1097/QAI. 0b013e3181bc0f10.

22. Wieland U, Brockmeyer NH, Weissenborn SJ, Hochdorfer B, Stucker M, Swoboda J, et al. Imiquimod treatment of anal intraepithelial neoplasia in HIV-positive men. Arch Dermatol. 2006;142(11):1438-44. doi:10.1001/archderm.142.11.1438.

23. Cranston RD, Hirschowitz SL, Cortina G, Moe AA. A retrospective clinical study of the treatment of high-grade anal dysplasia by infrared coagulation in a population of HIV-positive men who have sex with men. Int J STD AIDS. 2008;19(2):118-20. doi:10.1258/ijsa.2007.005665.

24. Morton CA, Birnie AJ, Eedy DJ. British Association of Dermatologists' guidelines for the management of squamous cell carcinoma in situ (Bowen's disease) 2014. Br J Dermatol. 2014;170(2):245-60. doi:10.1111/bjd.12766.

25. Goldstone SE, Johnstone AA, Moshier EL. Long-term outcome of ablation of anal high-grade squamous intraepithelial lesions: recurrence and incidence of cancer. Dis Colon Rectum. 2014;57(3):316-23. doi:10.1097/DCR.0000000000000058. 